\title{
The organization of classical satellite DNAs in human chromosomes: an approach using Alul and Taql restriction endonucleases
}

\author{
M. Nieddu, ${ }^{*}$ G. Pichiri, ${ }^{*}$ G. Diaz, ${ }^{\circ}$ R. Mezzanotte* \\ *Dip. di Scienze Applicate ai Biosistemi, Sezione di Biologia e Genetica; \\ ${ }^{\circ}$ Dip. di Citomorfologia, Cittadella Universitaria, Monserrato, Cagliari, Italy
}

(C)2003, European Journal of Histochemistry

Human classical satellite DNAs were used as probes to investigate the molecular mechanism(s) of Alul/Taql attack in situ on specific centromeric areas. The biochemical results obtained show that the majority of such highly repetitive DNAs are not solubilized from chromosomes, in spite of a cleavage pattern identical to that shown in naked genomic DNA digested with the same enzymes. Moreover, when digestion in situ with restriction enzymes precedes in situ hybridization, it is possible to observe an increased signal in the centromeres of some chromosomes as compared to that shown in standard undigested chromosomes and, on the other hand, hybridization labelling in centromeres which are difficult to detect by in situ hybridization using standard undigested chromosomes. Lastly, our results show that centromeric heterochromatin is not a homogeneous class in regard to organizational structure.

Key words: satellite DNA, human chromosomes, Alul, Taql

Correspondence: M. Nieddu, Dipartimento di Scienze Applicate ai Biosistemi, Sezione di Biologia e Genetica, Cittadella Universitaria, Monserrato, Cagliari, Italy.

Paper accepted on November 8, 2002

European Journal of Histochemistry 2003; vol. 47 issue 3 [Jul-Sept]: 209-214
A number of highly repetitive sequences are present in the centromeric/paracentromeric heterochromatin of human chromosomes and constitute about $13 \%$ of total genomic human DNA (see Tyler-Smith and Willard, 1993). Two main classes of such sequences exist: i) alphoid DNAs, which represent specific variants of a basic $170 \mathrm{bp}$ repeating unit and $\mathrm{ii)} \mathrm{three} \mathrm{major} \mathrm{highly} \mathrm{repetitive} \mathrm{sequences,} \mathrm{iso-}$ lated from bulk DNA by $\mathrm{CsSO}_{4}$ gradients and designated as classical satellite DNAs I, II and III, representing about $5 \%$ of the total human genome (Prosser et al, 1986). In turn, "each of the classical satellites I, II and III was found to contain, as a major component, a single family of repeated sequences. The three simple-sequence families have been called S1, S2 and S3 to differentiate them from these classical satellites, which also contain other repeated components" (Prosser et al, 1986).

On the other hand, the capability of restriction endonucleases (REs) to cleave specific base sequence targets in the DNA of cytological preparations is well-known and makes such enzymes powerful tools for producing chromosome banding as well as for detecting chromosomal localization of specific repetitive DNA fractions (Mezzanotte et al, 1983; Miller et al, 1983). In eukaryote organisms, highly repetitive sequences are known to be principally concentrated in particular chromosome regions such as centromeres. As a consequence, understanding molecular mechanism(s) accounting for RE-action in situ might furnish information regarding the organizational and functional role of such sequences. In this connection, it has recently been demonstrated that, even though the centromeric alphoid sequences of some human chromosomes are cleaved by either AluI or TaqI digestion in both naked DNA and the DNA of cytological preparations, DNA extraction from chromosomes and nuclei occurs only to a limited extent, and RE pre-treatment produces an increased signal when some alphoid DNAs are used as probes in fluores- 
cent in situ hybridization ( Nieddu et al, 1999).

To obtain further information on human centromeric organization and to complete the data obtained using alphoid DNAs (Nieddu et al, 1999), we carried out this work by employing sequences belonging to classical satellite DNAs as probes. Our results confirm that $\mathrm{RE}$ digestion in situ might vary FISH intensity centromeric signal but, on the other hand, show that this is not a general rule, due to the fact that each centromere represents a peculiar structural domain.

\section{Materials and Methods}

\section{Cytological experiments}

Human metaphase chromosomes were obtained from female peripheral blood lymphocytes of healthy donors, according to standard procedures ( Nieddu et al, 1999). Preparations were air dried for $24 \mathrm{~h}$ and subsequently digested with 30 units of either AluI or TaqI (Gibco BRL, Life Technologies) for $3 \mathrm{~h}$ at $37^{\circ} \mathrm{C}$, according to Mezzanotte et al. (1983). After RE treatment, DNA was isolated from $i)$ the material contained in the solution lying between the slide and the coverslip and ii) the material collected by carefully scraping the chromosome preparations on the slides with a sterilized razor blade. To avoid cross-contamination between the DNA contained in the solubilized material (soDNA) and that present in the DNA retained on the slides (re-DNA) after RE digestion in situ, slides were carefully rinsed using WLC buffer (10 $\mathrm{mmol} / \mathrm{L}$ Tris; $20 \mathrm{mmol} / \mathrm{L}$ EDTA; $10 \mathrm{mmol} / \mathrm{L} \mathrm{NaCl}$, $\mathrm{pH}$ 8.0), after collecting the solubilized material. Fluorescent in situ hybridization (FISH) was carried out on untreated chromosome preparations as well as on chromosomes previously treated with AluI or TaqI. The probes used in the present study, designated $\mathrm{S} 1, \mathrm{~S} 2$ and $\mathrm{S} 3$, belong to classical satellites I, II and III respectively and are single stranded oligonucleotides obtained according to Tagarro et al. (1994 a,b), by automated synthesis (Amersham Pharmacia Biotech). Their sequences are: $\mathrm{S}$ l: 5'TAT ACT GTA CAT AAA ATA TCA AAG TAC 3' (27-mer); S2: 5'TCG AGT CCA TTC GAT GAT 3' (18-mer); S3: 5'TCC ACT CGG GTT GAT T 3' (16mer). We stress that, due to the fact that S2 and S3 share the pentanucleotide ATTCC (Grady et al, 1992), we used the relative olinucleotides not containing such a sequence to prevent cross-hybridization (Tagarro et al, 1994 b). Oligonucleotides used for in situ hybridization were labelled at the $5^{\prime}$ end with biotin by Amersham Pharmacia Biotech. FISH was carried out according to Nieddu et al (1999), the stringency conditions being those used by Tagarro et al. (1994 a, b).

Control experiments were those carried out by incubating fixed chromosomes for the same time and temperature with the incubation buffer but without the enzyme used for digestion in situ.

For quantitative analysis, 24-bit RGB colour images were digitized using Image-Pro Plus (Media Cybernetics, Silver Spring, MD, USA). Preliminary measurements showed complete separation of $\mathrm{PI}$ and FITC signals into the red and green channels extracted from the RGB image. Chromosome axes were manually traced on PI outlines and then used to acquire longitudinal FITC profiles. Satellite DNA fluorescence intensity was evaluated as the difference between the centromeric peak and background FITC fluorescence of the chromosomes. Data were statistically analyzed using Statistica (StatSoft Inc, Tulsa, OK, USA). Differences between treated and untreated chromosomes were assessed by Student's t-test.

\section{Biochemical experiments}

Genomic DNA, purified from the same peripheral blood lymphocytes used in cytological experiments, was analysed by agarose-gel electrophoresis, according to Maniatis et al. (1982). Electrophoresis of either genomic DNA or the DNA belonging to the soand re-fractions (see Cytological Experiments), was carried out for $2 \mathrm{~h}$ at $100 \mathrm{~V}$, and DNA samples were subsequently transferred onto Hybond $\mathrm{N}+$ filters (Amersham). The same olinucleotide probes used in cytological experiments were employed for Southern blot hybridization, using a $3^{\prime}$-oligolabelling and detection system ( $E C L$, Amersham Life Science).

\section{Results}

\section{Cytological experiments}

FISH carried out using the consensus oligonucleotide sequences specific for classical satellite DNAs as probes shows standard localization (Figures $1 \mathrm{a}, d, g$ ), as already reported (Tagarro et al, 1994 a,b). However, in chromosomes predigested with AluI or TaqI, the labelling signal obtained using $\mathrm{SI}$ as a probe increases in the centromeric area of chromosomes 3 and 13, while no significant variation is found when the same measurement is 

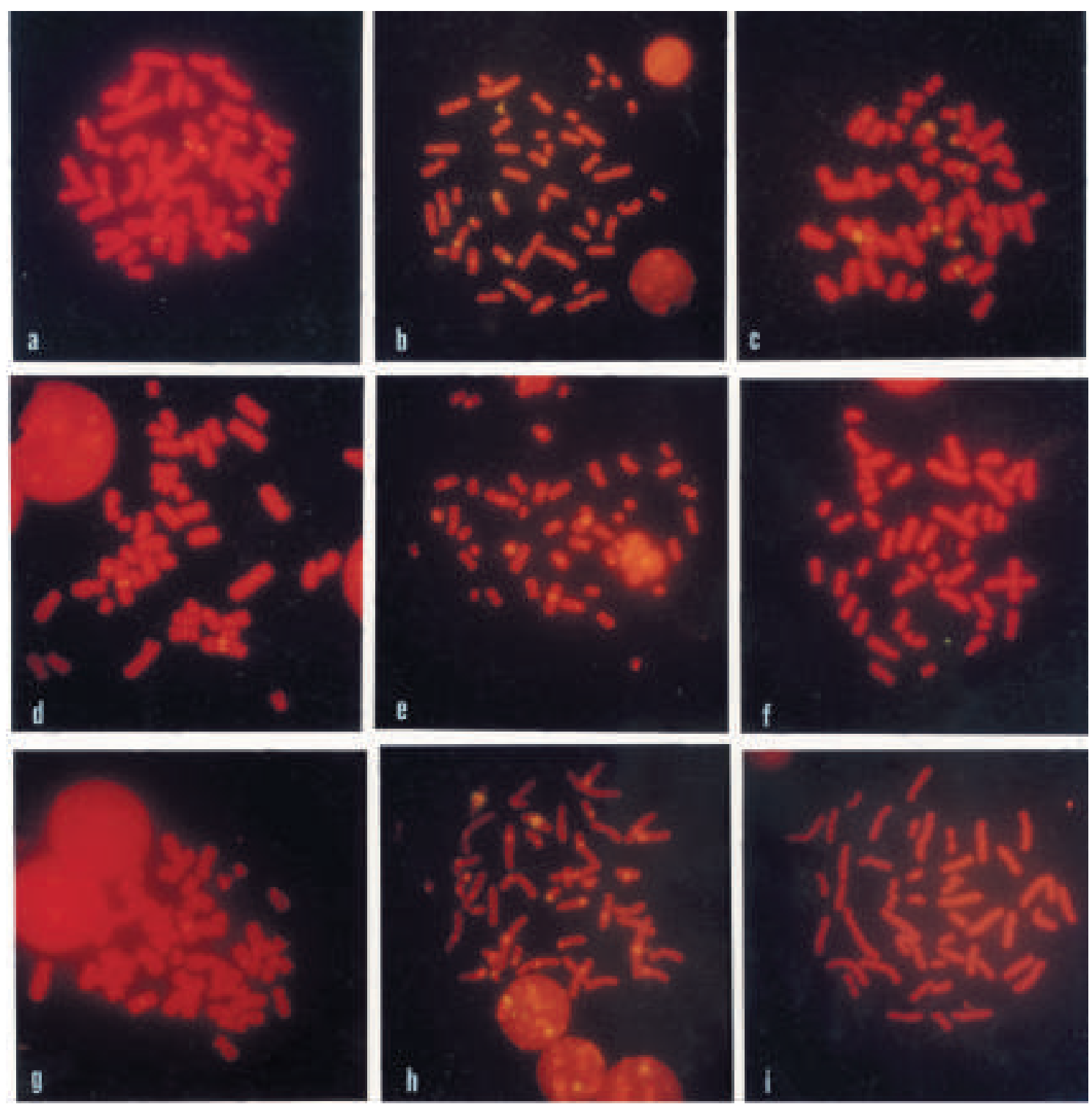

Figure 1. The in situ hybridization pattern using S1, S2 and S3 as probes (see Materials and Methods) in: standard, untreated preparations $(a, d, g)$; preparations pretreated with Alul $(b, e, h)$ and preparations pretreated with Taql (c, f, i). Note that hybridization signal fluorescence intensity is enhanced in some cases. In others, it is possible to observe the presence of hybridization sites which are not visible in control experiments (for details, see Results).

effected in the centromere of chromosome 4 (Figure $1 \mathrm{~b}, \mathrm{c}$; Figure 2). When FISH is effected using S2 as a probe, no significant difference in signal is found in the centromeres of chromosomes 1 and 16 after AluI pretreatment, while TaqI predigestion in situ results in the absence of any signal when FISH is carried out using either S2 or S3 as probes (Figure 1 e,f, i). However, when $\mathrm{S} 3$ is used as a probe after AluI treatment, it is possible to detect hybridization sites impossible to observe in standard preparations, in addition to an increase in signal intensity in areas such as chromosome 9 centromere.

Statistically relevant variations in digested as opposed to undigested chromosomes are summarized in Figure 2. 


\section{FITC fluorescence intensity (au)}

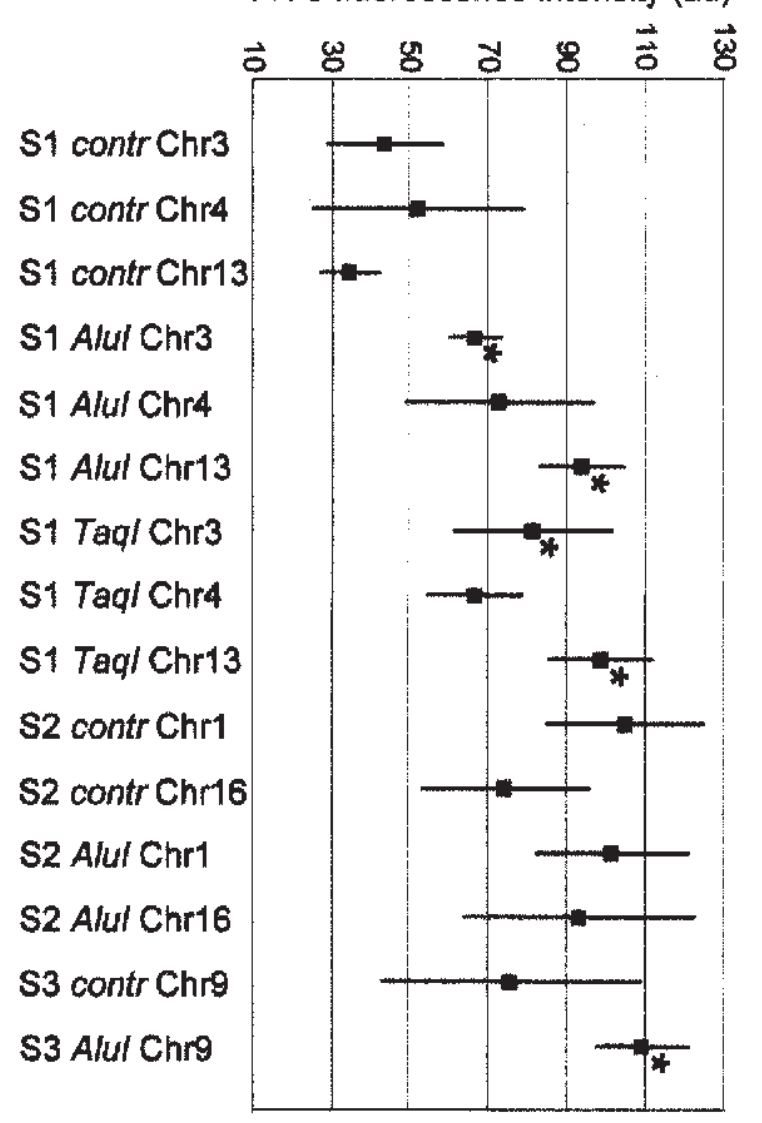

Figure 2. Plot showing the mean and standard deviations in FITC fluorescence intensity of classical human satellite DNAs. Statistically significant changes with respect to controls are labelled by asterisks.

\section{Biochemical experiments}

Before describing in detail the results relative to single sequences, let us stress that, in general, it is possible to observe that $\mathrm{i}$ ) the majority of $\mathrm{S} 1, \mathrm{~S} 2$ and S3 DNA is retained on the chromosomes after RE-digestion in situ, and ii) the cleavage pattern present in re-DNA fractions is identical to that shown by genomic DNA. In detail, S1 cleavage by either AluI or TaqI produces a hybridization ladder pattern of bands whose size ranges from about 3 to 1-2 $\mathrm{Kb}$ (Figure $3 \mathrm{a}$ ). Digestion of $\mathrm{S} 2$ sequences shows that AluI produces DNA fragments ranging from $23 \mathrm{~kb}$ to about $1500 \mathrm{bp}$, with the latter bands present in both so- and re-DNA fractions. TaqI acts so efficiently on S2 to produce a faint smear of fragments whose size ranges from 2000 to less than 500 bp (Figure 3b). The line containing genomic DNA does not show any hybridization signal possibly due to the greater TaqI cleavage efficiency in naked DNA as opposed to DNA part of fixed chrromosomes. In the case of S3 as well, it is possible to observe that AluI digestion induces hybridazion bands ranging from $23 \mathrm{~kb}$ to about $1800 \mathrm{bp}$, while it is possible to find a smear of DNA fragments only in the re-DNA fraction when $S 3$ is digested with TaqI (Figure 3 c).

\section{Discussion}

Our biochemical data on S1 show that i) only a limited number of $\mathrm{SI}$ sequences are cleaved by either AluI or TaqI, and ii) the DNA recovered after RE-digestion in situ is almost exclusively found in the re-fraction. These facts agree with the results reported by Frommer et al (1982), and represent a possible explanation for our cytological data. In fact, according to Nieddu et al. (1999), we postulate that the increased FISH signal observed in chromosomes 3 and 13 when $\mathrm{S} 1$ is used as a probe after AluI/TaqI digestion in situ is due to RE-cleavage followed by limited, if any, DNA extraction. This would produce the reorganization of centromeric/paracentromeric heterochromatin which, in turn, would permit an interaction between chromosomal DNA and DNA probe that is more efficient as compared to that occurring in untreated chromosomes. That heterochromatin reorganization might alter the accessibility of specific sequences in hybridization experiments would be indicated by the results found in chromosomes digested with AluI and subsequently treated by FISH using S3 oligomere as a probe. In this case, in fact, we found hybridization signal in a number of centromeres which do not show positive reaction in standard, undigested preparations. On the other hand, that RE-pretreatment might affect probe accessibility is also confirmed by the fact that when FISH is carried out after TaqI digestion in situ, no clear hybridization appears in centromeres though the majority of S3 DNA is retained on the slides, as shown in Figure 3c. This implies that FISH signal enhancement does not always occur after RE-digestion in situ. In fact, while TaqI pre-treatment results in an absence of signal with both S2 and S3 as probes, possibly due to the capability of this enzyme to extensively cleave such satellite DNAs (see also Moyzis et al., 1987; Vissel et al., 1992), FISH effected after AluI digestion reveals no significant variation in signal 

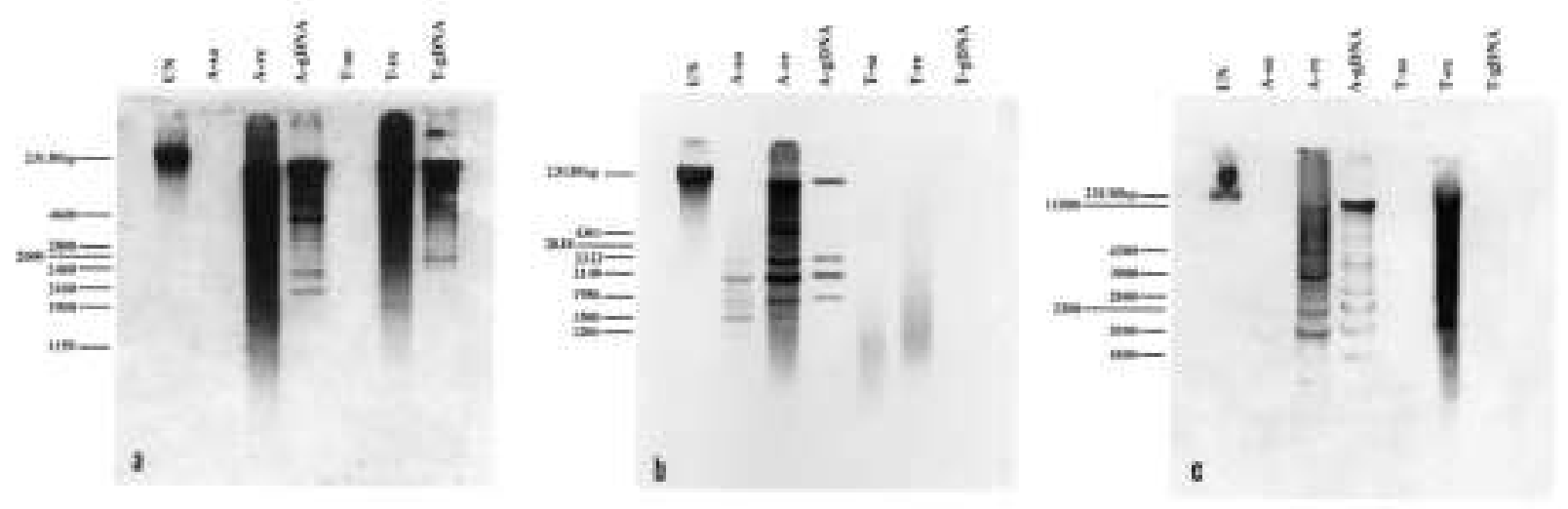

\begin{abstract}
Figure 3. Filter hybridization using human satellite DNAs S1(a), S2 (b) and S3 (c) respectively as probes. In detail, UN is undigested DNA; A-so and A-re are, respectively, solubilized DNA and DNA retained on the slides after Alul digestion in situ, while T-so and T-re are solubilized DNA and DNA retained on the slides after Taql digestion in situ. A-gDNA and T-gDNA are naked, genomic DNAs cleaved with Alul and Taql respectively. The size of specific hybridization bands is calculated on the basis of Ethidium Bromide stained electrophoretic pattern containing $\lambda$ DNA cleaved with HindIII or Pstl as markers (data not shown).
\end{abstract}

intensity in centromeric heterochromatin of chromosomes 1 and 16, as compared to undigested preparations. This is noteworthy if one considers that a similar attack, in terms of re-DNA fragment production (about 1200-200 bp in size), is effected by AluI and induces dramatic FISH signal enhancement in the chromosome 9 centromeric area. This would imply that RE attack on heterochromatic centromeric/paracentromeric areas is not always followed by structural changes which favour DNA hybridization in situ and, above all, that such heterochromatin does not constitute a homogeneous class of nucleoprotein organization, each centromere representing a peculiar structural domain.

Lastly, it is important to stress our results relative to the size of DNA fragments produced by cleavage in situ, directly related to RE-digestion efficiency. For instance, we found S2 DNA fragments of the same size (about 1200-1500 bp) in either so- or re-DNA fractions after cytological preparation treatment with AluI. This is noteworthy, since DNA solubilization is believed directly dependent on digestion fragment size, i.e. the larger the fragments, the more difficult it is to extract them (for a review, see Gosalvéz et al., 1997). Therefore, our data suggest that within each centromere, different domains exist which contain specific DNA sequences whose organization results in different RE-activity in situ.

\section{Acknowledgments}

The authors thank Mary Ann Groeneweg for checking the English style of the manuscript.

\section{References}

Frommer M, Prosser J, Tkachuk D, Vincent PC. Simple repeated sequences in human satellite DNA. Nucleic Acids Res 1982;10:54763.

Gosalvéz J, Lopez-Fernandez C, Goyanes V, Mezzanotte R. Chromosome differentiation using nucleases: an overview. In: Chromosomes Today, vol. 12. Henriques-Gil N, Parker JS, Puertas MJ, editors. Chapman \& Hall;1997. pp. 23-49.

Grady DL, Ratliff RL, Robinson DL, Mc Canlies EC, Meyne J, Moyzis RK. Highly repetitive DNA sequences are present at human centromeres. Proc Natl Acad Sci USA 1992;89:1695-9.

Maniatis T, Fritsch EF, Sambrook KJ. Isolation of high molecular weight, eukaryotic DNA from cells grown in tissue culture. Molecular Cloning: Cold Spring Harbor Laboratory Press; New York. 1982, p. 280-1.

Mezzanotte R, Ferrucci L, Vanni R, Bianchi U. Selective digestion of human metaphase chromosomes by AluI restriction endonuclease. $J$ Histochem Cytochem 1983;31:553-6.

Miller DA, Choi YC, Miller OJ. Chromosome localization of highly repetitive human DNAs and amplified ribosomal DNA with restriction enzymes. Science 1983;219:395-7.

Moyzis RK, Albright KL, Bartholdi MF, Cram LS, Deaven LL, Hildebrand $\mathrm{CE}$, et al. Human chromosome-specific repetitive DNA sequences: novel markers for genetic analysis. Chromosoma 1987; 95:375-86

Nieddu M, Rossino R, Pichiri G, Rocchi M, Setzu MD, Mezzanotte R. The efficiency of in situ hybridization on human chromosomes with alphoid DNAs is enhanced by previous digestion with AluI and TaqI. Chromosome Res 1999; 7:593-602.

Prosser J, Frommer M, Paul C, Vincent PC. Sequence relationships of three human satellite DNAs. J Mol Biol 1986;187:145-55.

Tagarro I, Wiegant J, Raap AK, Gonzalez-Aguilera JJ, FernandezPeralta AM. Assignement of human satellite 1 DNA as revealed by fluorescent in situ hybridization with oligonucleotides. Hum Genet 1994; 93:125-8.

Tagarro I, Fernandez-Peralta AM, Gonzalez-Aguilera JJ. Chromosomal localization of human satellite 2 and 3 by a FISH method using oligonucleotide as probes. Hum Genet 1994;93:383-8.

Tyler-Smith C, Willard, HF. Mammalian chromosome structure. In Current Opinion in Genetics and Development. vol. 3. Current Biology Ltd., p. 390-7.

Vissel B, Nagy A, Choo KHA. A satellite III sequence shared by human chromosomes 13,14 and 21 that is contiguous with a satellite DNA. Cytogenet Cell Genet 1992;61:81-6. 